\title{
The emergence of multimodal imaging methods for real-time nanoscopy
}

\author{
Cyril Petibois
}

Published online: 9 June 2011

(C) Springer-Verlag 2011

\section{"High-performance imaging techniques, notably those based on synchrotron radiation sources, are defining a new analytical field named real-time nanoscopy." (C. Petibois)}

The field of microscopy has evolved from simple brightfield on-axis imaging to that of nonvisible and confocal-based technologies. Bright-field imaging is a resolution-limited technique that uses on-axis illumination and imaging to form the image, typically with visible light. Confocal microscopy allows improved contrast and resolution as well as an extremely small depth of focus. By combining confocal with nonvisible techniques, researchers can now look through certain materials to see inside complex objects. The development of imaging techniques has continuously shaped the field of life sciences, covering different scales for ex vivo and in vivo applications, as well as timeresolved acquisitions for biochemical and physiological analyses. Material sciences have also benefited from imaging technique enhancements, providing access to the nanostructure of complex alloys, showing the organization of polymers, etc. More globally, imaging techniques allow complex internal organizations, interfaces between structuring compartments, elemental or molecular distributions, etc. to be revealed. Imaging techniques give access to various types of information from analytical specimens, and depending on the interaction between the

Published in the special issue Imaging Techniques with Synchrotron Radiation with Guest Editor Cyril Petibois.

\section{Petibois $(\square)$}

University of Bordeaux,

CNRS UMR 5248 CBMN Allée de Saint-Hillaire,

F33600 Pessac-Cedex, France

e-mail: c.petibois@cbmn.u-bordeaux.fr sample and the waves or particles used to probe it, elemental, chemical, molecular, and morphological characterization is achieved. Combining the use of several techniques on the same sample was implemented a few decades ago, notably for the development of the socalled molecular imaging applications, mostly using Xray computed tomography (CT) for morphological rendering of the sample, and MRI or PET to obtain chemical information. The result is the localization of the chemical information in its $3 \mathrm{D}$ environment. This is different from multiple-imaging techniques, which use a duplication of the same phenomenon (e.g., multiphoton) or can be applied as a spectroscopy (IR, Raman, X-ray fluorescence) to extract several parameters from the same interaction with sample contents. Nevertheless, multipleimaging and multimodal imaging techniques have now acquired the status of standards in different scientific fields. However, other initiatives have been proposed and some have already successfully enlarged the panel of possible applications. Three-dimensional imaging techniques have improved our sample characterization approach since they have opened the way to quantitative analyses of small objects and they have definitively pushed science into the nanoworld. Nano-objects are present in daily life at all levels, in building materials, foods, clothes, and all our familiar objects. We can cite the mineral and synthetic fibers we breathe, the pesticides we eat or drink, the nanoparticles in our cosmetics, etc. Thus, many specimens interact with the cells and tissues of our body. Imaging these interactions clearly requires a multimodal imaging approach, with in vivo access to the information if the technique becomes a diagnostic tool in clinics. X-ray CT is not yet capable of nanometer resolution in vivo for humans, but it is becoming feasible for cells or even small animals. Thus, 
fundamental investigation of diseases using living models has now developed. X-ray fluorescence spectroscopy is also achieving the status of a 3D imaging technique, thus offering the possibility of obtaining the spatial distribution of elements in microsized objects. Near-field techniques are getting closer to nanoscale resolutions (below $100 \mathrm{~nm}$ ) and can thus resolve nanoobjects at the surface of microsystems. Dedicated methods have been proposed to couple these techniques for application on the same sample, and sometimes for concurrent image acquisitions. Therefore, multimodal imaging is defining the concept of "real-time nanoscopy" and the performances of synchrotron radiation (SR) sources are showing the way for future laboratory routine equipment. This concept is a new field for analytical sciences in the sense that it changes our way of studying matter. Real-time nanoscopy concerns all techniques now able to image samples below their former limits (e.g., diffraction limit for IR spectroscopy, ex vivo only for X-ray fluorescence or coherent antiStokes Raman spectroscopy) and thus allows the use of the techniques to be extended from their previously reserved domain to other scientific fields. Being able to manage critical limitations, such as the action of ionization (X-ray and UV techniques) or heating (Raman technique) processes on samples, is also removing a major restriction for multimodal imaging applications.

There is no doubt that SR-based imaging techniques will continue to enlarge our knowledge of complex systems and raise new fundamental questions on matter organization and function in many different fields. The ITSR workshop in Suzhou, China, 6-9 November 2010, outlined this perspective. Shifting from biological to material sciences, some examples included (1) 3D characterization of microvascularization foci to reveal acupuncture points; (2) the development of the combination of X-ray CT and fluorescence spectroscopy for characterization of a single cell or yeast; (3) accessing the internal organization of organs in insects to refine the tree of evolutionary development; (4) revealing chemical and morphological interactions between natural inorganic materials and living systems by combining spectral, morphological, and surface analyses of the same sample; (5) characterization of solid fuel cell electrode performances based on their microstructure and morphology; (6) revelation of intrinsic multiscale inhomogeneity in superconductor systems at nanoscale phase separation.

The Suzhou workshop has thus shown that real-time nanoscopy is a new emerging field of imaging technology. New SR-based equipment or beamlines are now foreseen, combining X-ray phase contrast and fluorescence approaches for morphological/elemental analysis of microsized objects, or combining two SR sources for the development of time resolved X-ray and IR concurrent analysis.

The next ITSR workshop will be held in Bordeaux, 2427 September 2011 (see http://www.cbmn.u-bordeaux.fr/ ESF-ITSR/index-ITSR.html) and will precede an ESF workshop on "New high-resolution multimodal techniques for the imaging of living systems" to be held on 28-30 September 2011 (see http://www.cbmn.u-bordeaux.fr/ESFITSR/index-ESF.html; for further details, please contact C. Petibois via c.petibois@cbmn.u-bordeaux.fr).

Cyril Petibois is Assistant Professor of Biochemistry at the University of Bordeaux, France, and is involved in the development of imaging methods for analysis of biosamples (CNRS UMR 5248 research unit; "Spectro-imaging of Biosystems" group). His research interest is to develop multimodal imaging methods for full characterization of biosamples, from synthetic models to individual cells and small animals, combining elemental, morphological, molecular, and chemical techniques. The laboratory is also engaged in the development of highperformance imaging methods using synchrotron radiation, notably using IR and X-ray beamlines at major European and Asian synchrotron radiation facilities. 